\title{
Palatability and pharmacokinetics of flunixin when administered to sheep through feed
}

Danila Marini, Joe Pippia, Ian G Colditz, Geoff N Hinch, Carol J Petherick, Caroline Lee

Applying analgesics to feed is a potentially easy method of providing pain-relief to sheep and lambs that undergo painful husbandry procedures. To be effective, the medicated feed needs to be readily accepted by sheep and its consumption needs to result in therapeutic concentrations of the drug. In the present experiment, pelleted feed was supplemented with flunixin $(4.0 \mathrm{mg} / \mathrm{kg}$ live weight) and offered to eight sheep. To test the palatability of flunixin, the individually penned sheep were offered normal feed and feed supplemented with flunixin in separate troughs for two consecutive days. A trend for a day by feed-type (control versus flunixin supplemented) interaction suggested that sheep may have had an initial mild aversion to pellets supplemented with flunixin on the first day of exposure, however, by on the second day there was no difference in consumption of normal feed and feed supplemented with flunixin. To test pharmacokinetics, sheep were offered $800 \mathrm{~g}$ of flunixin supplemented feed for a $12 \mathrm{~h}$ period. Blood samples were taken over $48 \mathrm{~h}$ and plasma drug concentrations were determined using ultra-high-pressure liquid chromatography, negative electrospray ionisation and tandem mass spectrometry. The mean \pm S.D. time required to reach maximum concentration was $6.00 \pm 4.14 \mathrm{~h}$ and ranged from 1 to $12 \mathrm{~h}$. Average maximum plasma concentration was $1.78 \pm 0.48 \mu \mathrm{g} / \mathrm{mL}$ and ranged from 1.61 to $2.80 \mu \mathrm{g} / \mathrm{mL}$. The average half-life of flunixin was $7.95 \pm 0.77 \mathrm{~h}$ and there was a mean residence time of $13.62 \pm 1.17 \mathrm{~h}$. Free access to flunixin supplemented feed enabled all sheep to obtain inferred therapeutic concentrations of flunixin in plasma within $6 \mathrm{~h}$ of starting to consume the feed. Provision of an analgesic in feed may be an alternative practical method for providing pain relief to sheep. 
1

Palatability and pharmacokinetics of flunixin when administered to sheep through feed ${ }^{1}$

2

3 D. Marini1 ${ }^{1,2, *}$ J. Pippia $^{3}$, I. G. Colditz ${ }^{1}$, G. Hinch ${ }^{2}$, J. C. Petherick ${ }^{4}$ and C. Lee ${ }^{1}$

$4 \quad{ }^{1}$ CSIRO, Agriculture, Armidale, New South Wales, Australia

52 The University of New England, Armidale, New South Wales, Australia

$6 \quad 3$ PIA PHARMA Pty Ltd, Gladesville, New South Wales, Australia

$7{ }^{4}$ The University of Queensland, Brisbane, Queensland, Australia

9

10

11

12

13

14

15

16

17

18

*Danila Marini: CSIRO, Agriculture, New England Highway, Armidale, New South Wales, Australia, 2350, (danila.marini@csiro.au) 


\section{ABSTRACT:}

Applying analgesics to feed is a potentially easy method of providing pain-relief to sheep and lambs that undergo painful husbandry procedures. To be effective, the medicated feed needs to be readily accepted by sheep and its consumption needs to result in therapeutic concentrations of the drug. In the present experiment, pelleted feed was supplemented with flunixin $(4.0 \mathrm{mg} / \mathrm{kg}$ live weight) and offered to eight sheep. To test the palatability of flunixin, the individually penned sheep were offered normal feed and feed supplemented with flunixin in separate troughs for two consecutive days. A trend for a day by feed-type (control versus flunixin supplemented) interaction suggested that sheep may have had an initial mild aversion to pellets supplemented with flunixin on the first day of exposure, however, by on the second day there was no difference in consumption of normal feed and feed supplemented with flunixin. To test pharmacokinetics, sheep were offered $800 \mathrm{~g}$ of flunixin supplemented feed for a $12 \mathrm{~h}$ period. Blood samples were taken over $48 \mathrm{~h}$ and plasma drug concentrations were determined using ultra-high-pressure liquid chromatography, negative electrospray ionisation and tandem mass spectrometry. The mean \pm h. Average maximum plasma concentration was $1.78 \pm 0.48 \mu \mathrm{g} / \mathrm{mL}$ and ranged from 1.61 to 2.80 $\mu \mathrm{g} / \mathrm{mL}$. The average half-life of flunixin was $7.95 \pm 0.77 \mathrm{~h}$ and there was a mean residence time of $13.62 \pm 1.17 \mathrm{~h}$. Free access to flunixin supplemented feed enabled all sheep to obtain inferred therapeutic concentrations of flunixin in plasma within $6 \mathrm{~h}$ of starting to consume the feed. Provision of an analgesic in feed may be an alternative practical method for providing pain relief to sheep. 


\section{INTRODUCTION}

45 Flunixin meglumine is a potent non-steroidal anti-inflammatory drug (NSAID) that is commonly used in veterinary medicine for its anti-inflammatory, analgesic and antipyretic properties. Like

47 other NSAIDs, flunixin reduces inflammation by inhibiting cyclooxygenase and, in turn, decreasing the production of prostaglandins (Cheng et al., 1998b), which are important inflammatory mediators. Flunixin is known to be effective at relieving pain in various domesticated species such as horses (Keegan et al., 2008; Toutain et al., 1994) and cattle (Currah et al., 2009) and is currently registered for use for these animals in the USA, Europe and Australia. Although flunixin has also been shown to be effective for pain relief in sheep (Paull et al., 2007; Welsh 1995), there are currently no NSAIDs registered in Australia for use in sheep. Pain relief can be impractical and costly to administer to livestock raised in extensive systems

55 due to the necessity for repeated application over time and the limited availability of registered drugs (Lizarraga \& Chambers, 2012). A potential practical method of providing pain relief is

57 through oral administration, allowing farmers to either provide NSAIDs as a drench or through feed in the form of granules or a liquid formulation. It is known that the rumen can decrease the bioavailability of NSAIDs if they are administered orally (Mosher et al., 2012; Odensvik 1995). In previous work to counteract the reduced bioavailability when administering NSAIDs orally to

61 cattle the dose given was double compared with that recommended for parenteral administration

62 (Coetzee et al., 2012). 
63 Incorporation of flunixin to an animal's diet could possibly elicit a neophobic response or

64 reduced feed intake if flunixin is unpalatable. Therefore the objectives of this study were 1) to

65 test the palatability of flunixin and 2) determine the pharmacokinetics of flunixin in sheep

66 plasma when feed supplemented with flunixin was offered. We hypothesised that all sheep

67 would achieve therapeutic concentrations of flunixin in plasma when consuming feed

68 supplemented with flunixin.

MATERIALS AND METHODS

\section{Experimental animals}

Nine, 2-year-old, maiden Merino ewes with mean live weight of $38.8 \pm 2.83 \mathrm{~kg}$ (mean \pm S.D.) were used in this study. Sheep were clinically healthy at the time of the study. Upon entry to the

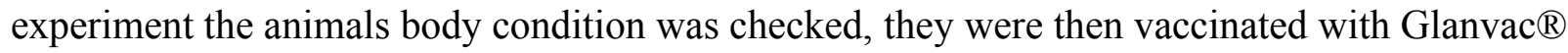
6S B12 (Zoetis Animal Health, Australia) and drenched with Firstmectin (Virbac, Milperra, NSW, Australia), Flukazole C (Virbac, Milperra, NSW, Australia) and Rycozole (Novartis, North Ryde, NSW, Australia) at the manufacturers' recommended dose rates. Following vaccination and drenching the sheep were then monitored daily for any signs of ill health, such as behavioural and respiratory changes. There was a month between drenching treatments and the pharmacokinetic experiment. The sheep were housed in individual pens in a covered shed which was open on the North face and were in close proximity to allow visual and social interaction with other experimental animals. Animals were fed a complete pelleted ration (Ridley Agriproducts, Australia; $17 \%$ crude protein dry matter; $9.04 \mathrm{MJ} / \mathrm{kg}$ dry matter). During acclimation, sheep were offered a small excess of feed over their previous day's intake (between $800-1000 \mathrm{~g}$ ) supplemented with $100 \mathrm{~g}$ of oaten chaff daily so that some residual feed was left at 
85 the end of each day. Water was also provided ad-libitum. The experiment was undertaken at

86 CSIRO's FD McMaster Laboratory, Armidale, New South Wales (NSW). The protocol and

87 conduct of the experiment was approved by The CSIRO Armidale Animal Ethics Committee

88 under the NSW Animal Research Act, 1985 (ARA 14/01).

89

90

91

92

93

94

95

96

97

98

99

100

101

102

103

104

105

106

\section{Palatability test}

One week prior to the start of the experiment, each animal was acclimatised to eating from two troughs within its pen and daily feed intake was monitored. The palatability test ran over 2 days; in the morning sheep were offered feed in two troughs, one containing $2 \mathrm{~kg}$ of the standard pelleted ration and the other containing $2 \mathrm{~kg}$ of the same standard pelleted diet supplemented with $20 \mathrm{~mL}$ (300 mg) of liquid flunixin (Flunixin Oral solution, 15 mg/mL, Pia Pharma Pty Ltd, Gladesville, NSW, Australia). The amount of flunixin added per kg of feed was equivalent to an approximate single dose for the live weight of each ewes (i.e. eating $1 \mathrm{~kg}$ of the supplemented feed would deliver 1 dose at $4 \mathrm{mg} / \mathrm{kg}$ body weight). The feed was prepared each morning by putting the liquid flunixin onto the pellets and thoroughly mixing them together in the trough; even incorporation of the liquid was characterised by the change in colour of the pellets.

Following flunixin application the trough did not appear to be wet and there was no free liquid present at the bottom of the trough. Both troughs were placed into the pen simultaneously and the location of the trough containing flunixin supplemented feed was alternated for the second day of testing.

\section{Pharmacokinetic protocol}

After the palatability test, the ewes were kept in a paddock for a 2 -week flush-out period. They were then returned to the same individual pens that were used for the palatability test, 1 week 
107 prior to the beginning of the pharmacokinetic experiment. The sheep were again fed the 108 complete pelleted ration ad libitum supplemented with $100 \mathrm{~g}$ of oaten chaff once a day. The day

109 prior to supplementation of feed with flunixin, sheep were weighed and had the wool clipped

110 from their necks. To allow for intensive blood sampling, catheters were inserted aseptically in

111 the left jugular vein using a $12 \mathrm{G}$ catheter needle to puncture the vein. A piece of catheter tubing

112 was then threaded through the needle and then, to ensure the catheter was inserted correctly, the

113 line was flushed with heparinised saline and then liquid withdrawn until blood was seen flowing.

114 Catheters were then re-flushed with heparinised saline. The catheter needle was removed and the 115 line was sealed with a three-way tap adaptor containing a luer lock syringe port. The line was 116 secured to the animal at the exit point with Elastoplast tape, the remaining catheter tubing was

117 then encased in $7.5 \mathrm{~cm}$ wide Elastoplast bandage which was gently wrapped around the sheep's 118 neck.

119 On the day of the study, sheep were offered $800 \mathrm{~g}$ of feed containing a dose of flunixin (at a rate 120 of $4.0 \mathrm{mg} / \mathrm{kg}$ live weight) adjusted for each animal's body weight. Flunixin was added to feed as 121 described for the palatability test. The first sheep was presented with the flunixin supplemented 122 feed at $0700 \mathrm{~h}$ and the remaining sheep were given their feed at 2 min intervals thereafter. Blood 123 samples $(10 \mathrm{~mL})$ were collected before the flunixin supplemented feed was offered $(0 \mathrm{~h})$ and at $1245,10,15,20,30,45 \mathrm{~min}$ and $1,2,4,6,8,12,24,36,48 \mathrm{~h}$ relative to the time each sheep was 125 first observed to have consumed some of the supplemented feed. Prior to the collection of each 126 blood sample, $2 \mathrm{~mL}$ of blood was withdrawn from the catheter and discarded to ensure that fresh 127 blood was collected. Blood samples were centrifuged $(2000 \times \mathrm{g})$ and the separated plasma 128 collected and frozen at $-20^{\circ} \mathrm{C}$. Residual feed remaining in the trough was weighed at each blood 129 sampling time point until $12 \mathrm{~h}$ post-initial ingestion. 


\section{Plasma flunixin concentration determination}

131 Plasma samples were transported frozen to Pia Pharma Pty Ltd, Gladesville, NSW for flunixin

132 concentration determination using ultra-high-pressure liquid chromatography, negative

133 electrospray ionisation and tandem mass spectrometry (UHPLC/ -ve ESI MS/MS). Each plasma

134 sample was thawed to room temperature on the day of analysis. For determination, a $250 \mu \mathrm{L}$

135 aliquot of each plasma sample was dispensed into a $2 \mathrm{~mL}$ polypropylene centrifuge tube.

136 Flunixin-d3 internal standard (50 $\mu \mathrm{L}$ of $2.0 \mu \mathrm{g} / \mathrm{mL}$ flunixin-d3) was added and the sample mixed

137 gently prior to the addition of $350 \mu \mathrm{L}$ acetonitrile. The sample was vortexed (1 min) and

138 centrifuged (13000 $\mathrm{rpm} / 5 \mathrm{~min})$ to remove any sediment. Type 1 water for UHPLC applications

$139(0.5 \mathrm{~mL})$ was then added to the extract and the mixture was filtered through a $0.45 \mu \mathrm{m}$ filter prior

140 to determination. An aliquot of sample extract $(5 \mu \mathrm{L})$ was injected into an Eksigent ${ }^{\circledR}$ Ekspert ${ }^{\mathrm{TM}}$

141 ultraLC 100-XL Liquid Chromatograph fitted with a Supelco Ascentis ${ }^{\circledR}$ Express 50 x $2.1 \mathrm{~mm}$,

$1422.7 \mu \mathrm{m}$ analytical column maintained at $40{ }^{\circ} \mathrm{C}$. A gradient elution program, based on a

143 combination of $0.1 \%$ formic acid and acetonitrile as mobile phase constituents operating at 0.4

$144 \mathrm{~mL} \mathrm{~min}-1$, resolved flunixin and flunixin-d3 (retention time of $2.5 \mathrm{~min}$ ) from matrix

145 interferences and endogenous sample components. The identity of peaks was predicted using an

146 AB Sciex API 3200 triple-quadrupole mass spectrometer interfaced with the liquid

147 chromatograph. The detector was configured with a proprietary turbo V source for desolvation

148 and operated in negative electrospray ionisation mode $(-4500 \mathrm{~V})$, desolvation temperature 550

$149{ }^{\circ} \mathrm{C}$, for optimum analyte selectivity and sensitivity. The transitions for flunixin and flunixin-d 3

150 were $295.1 \rightarrow 191.0$ and $298.2 \rightarrow 254.0$ respectively.

151 Matrix matched calibration standard solutions of flunixin were prepared at increasing

152 concentrations between 10 and $4000 \mathrm{ng} / \mathrm{mL}$ in plasma from animals prior to treatment. The 
153 calibration curve was prepared by plotting the nominal flunixin concentration ( $\mathrm{x}$ axis) against the

154 determined peak area ratio of flunixin and flunxin-d3 for each calibrator. A correlation co-

155 efficient (r) greater than 0.99 was required for the calibration curve to be used for quantitative

156 purposes. Analyte concentrations were calculated using the peak area ratio of flunixin detected in

157 each sample relative to the corresponding flunixin-d3 internal standard, and the regression

158 equation of the calibration curve.

159 Method accuracy and precision were monitored with the inclusion of fortified quality control

160 samples. Four plasma samples containing flunixin concentrations of 13.1, 328.5, 1314.1, 3942.3

$161 \mathrm{ng} / \mathrm{mL}(\mathrm{n}=3)$ were prepared on the day of the analysis. The mean percentage of accuracy was

$16290.8 \%$ at lower limit of quantification (LLOQ) and $102.9-111.6 \%$ at all other concentrations.

163 The coefficient of variation at LLOQ was 2.9\%, and 1.3-3.1\% at other concentrations. Quality

164 control data were acceptable.

165 Statistics

166 Palatability data were analysed with R-Project (R, Boston, Massachusetts) using nlme package

167 (Pinheiro et al., 2015) to perform a linear mixed model analysis. Fixed effects included feed type

168 (flunixin present or absent), day (1 or 2), and location of flunixin supplemented feed trough (left

169 or right) and the interaction of feed type by day. Sheep number was fitted as a random effect.

170 Results are presented as mean \pm S.D.. Data were tested for normality using the Shapiro-Wilk

171 test. $\mathrm{P}<0.05$ was considered as statistically significant.

\section{Pharmacokinetic analysis}

173 Pharmacokinetic modelling of flunixin in plasma was performed using an open source

174 pharmacokinetic program (PK Solver, China Pharmaceutical University, Nanjing, Jiangsu, 
175 China) (Zang et al., 2010). Using non-compartmental analysis, the maximum flunixin

176 concentration (Cmax) in plasma, the time required to reach $\mathrm{Cmax}$ (Tmax), mean residence time

177 (MRT) and elimination half-life (t1/2) were determined for each animal. The area under the

178 concentration vs. time curve $(\mathrm{AUC} 0-\mathrm{t})$ was calculated using the linear trapezoidal rule.

179 Pharmacokinetic parameters were estimated for each animal from which mean values \pm S.D.

180 were calculated.

\section{RESULTS}

\section{Palatability}

183 One ewe was excluded from data analysis as she did not consume any of the feed containing 184 flunixin on either day. Location of the different feeds (left or right trough) had no effect $(\mathrm{P}=$ 185 0.81) on the amount of each feed (flunixin supplemented versus control) that was consumed. 186 Although there was no main effect of feed type across days $(\mathrm{P}=0.10)$, a trend was observed for 187 the day by feed type interaction $(\mathrm{P}=0.08)$. On day 1 , animals consumed on average $551.14 \pm$ $188446.68 \mathrm{~g}$ more of the control feed than the flunixin supplemented feed $(\mathrm{P}=0.02)$. Whereas on day 2 there were no differences observed in the consumption of control feed and feed supplemented with flunixin $(\mathrm{P}=0.95)$. On day 2 , consumption of control feed decreased on average by $489.79 \pm 468.53$ from the quantity consumed on day $1(\mathrm{P}=0.03)$. Consumption of

192 feed supplemented with flunixin was comparable on days 1 and $2(\mathrm{P}=0.73$, Table 1$)$.

\section{Pharmacokinetics}

194 The sheep took between 8 and $12 \mathrm{~h}$ to consume the total $800 \mathrm{~g}$ of flunixin supplemented feed on 195 offer. Most of the sheep spread meals throughout the day except for ewe 466 who ate $350 \mathrm{~g}$ of

196 feed in the first 5 min and ewe 627 who consumed $332.5 \mathrm{~g}$ in the last $4 \mathrm{~h}$ of the $12 \mathrm{~h}$ period. 
197 Flunixin was absorbed rapidly, all sheep had detectable plasma concentrations $(>20 \mathrm{ng} / \mathrm{mL})$ at

$19810 \mathrm{~min}$ after initial consumption of supplemented feed with the exception of one animal (ewe

199 627), who only ate $21.5 \mathrm{~g}$ of feed in the first $10 \mathrm{~min}$.

200 All sheep started to eat within a few minutes after the provision of feed. There was large

201 variability between sheep in the amount of feed that was consumed at each time-point (Table 2).

202 When animals had free access to feed, the majority of sheep (7 out of 8) achieved plasma

203 flunixin concentrations above $1.0 \mu \mathrm{g} / \mathrm{mL}$ within $2 \mathrm{~h}$ of starting to consume the supplemented

204 feed, with maximum concentrations (between 1.33 and $2.80 \mu \mathrm{g} / \mathrm{mL}$ ) being reached on average

205 by $6 \mathrm{~h}$. Flunixin concentration time curve (mean \pm S.D.) in all sheep plasma over a period of $48 \mathrm{~h}$

206 is shown in Figure 1. This led to a large variability in the Tmax, which ranged from 1 to $12 \mathrm{~h}$.

207 The Cmax average was $1.78 \pm 0.48 \mu \mathrm{g} / \mathrm{mL}$ and the flunixin meglumine plasma $\mathrm{t}_{1 / 2}$ was $7.95 \pm$ $2082.19 \mathrm{~h}$ (Table 3).

\section{DISCUSSION}

211 Concentrations measured in this study were somewhat lower compared with those reported in

212 our previous study (Marini et al., 2015) where flunixin concentration in plasma reached values

213 between $2.6-4.1 \mu \mathrm{g} / \mathrm{mL} 2 \mathrm{~h}$ after a single oral dose $(4 \mathrm{mg} / \mathrm{kg})$ in sheep. Reports of therapeutic

214 concentrations of flunixin in farm animals are limited, however, Toutain et al., (1994) reported

215 therapeutic effects in horses when plasma concentrations reached $0.2-0.9 \mu \mathrm{g} / \mathrm{mL}$. The results of

216 the current study suggest that the plasma flunixin concentrations achieved following

217 consumption of supplemented feed may be within the therapeutic range for sheep. 
218 Although displaying an initial (day 1) preference for control pelleted feed over flunixin-

219 supplemented feed, there was no overall feed preference effect observed. The initial preference

220 of control pelleted feed may have been due to the novelty of the odour or flavour of flunixin.

221 Odour and flavour help sheep distinguish different types of feed and they are more likely to eat

222 novel feeds that contains some familiar flavours (Hinch et al., 2004; Launchbaugh et al., 1997).

223 Sheep are known to avoid novel feed types for several days before they start to consume it

224 (Chapple et al., 1987). Adding flunixin to a feed with which the ewes were familiar, may have

225 reduced any neophobia. With the exception of one ewe who did not consume any feed

226 supplemented with flunixin over the two days, the intake of supplemented and control feeds was

227 similar on the second day of testing.

228 In sheep, the pharmacokinetics of flunixin has been investigated following intramuscular and

229 intravenous administration (Cheng et al., 1998a; Welsh et al., 1993). When administered

230 intravenously, the elimination half-life of flunixin meglumine has been reported to be $2.48 \mathrm{~h}$

231 (Cheng et al., 1998a) and $3.83 \mathrm{~h}$ (Welsh et al., 1993). The elimination half-life observed in the

232 current study (following oral administration) was longer (7.95 $\pm 2.19 \mathrm{~h})$. Differences were also

233 observed for the MRT of flunixin following intravenous versus oral administration, with MRT in

234 plasma being $3.20 \pm 0.18 \mathrm{~h}$ (Cheng et al., 1998a) compared with $13.59 \pm 3.31 \mathrm{~h}$ in the current

235 study. When flunixin is administered intramuscularly and intravenously it is typically given as a

236 bolus dose, which permits a uniform pattern of absorption and elimination to occur. The longer

237 half-life and mean retention time observed in this study is likely due to animals consuming their

238 dose of flunixin over an extended period of time, rather than as a bolus. The AUC observed in

239 the current study $\left(37.62 \pm 4.77 \mu \mathrm{g} / \mathrm{mL}^{*} \mathrm{~h}\right)$ was similar to that reported by Cheng et al., (1998a) 
$240\left(30.61 \pm 3.41 \mu \mathrm{g} / \mathrm{mL}^{*} \mathrm{~h}\right)$. It is probable that our higher AUC was due to the higher dose rate used

241 in our study.

242

243 The pharmacokinetics of orally administered flunixin has been studied in goats (Königsson et al.

244 2003), horses (Pellegrini-Masini et al., 2004; Welsh et al., 1992) and cattle (Odensvik, 1995).

245 Following oral administration of a bolus dose in the absence of feed in these species, flunixin is

246 absorbed rapidly and concentrations can still be detected up to $30 \mathrm{~h}$ after administration

247 (Königsson et al., 2003; Odensvik, 1995). Horses that had ad libitum access to hay following the

248 oral administration of flunixin had a slower absorption of flunixin and a lower Cmax although

249 concentrations of flunixin in plasma were maintained for longer when animals had access to feed

250 compared with when they were fasted (Welsh et al. 1992). The AUC was not significantly

251 different when animals were fasted or non-fasted, suggesting that the absorption of flunixin is

252 not affected by the presence of feed. In the current study, flunixin was found to be absorbed

253 rapidly when consumed with feed, with detectable levels present within 10 min in sheep that

254 consumed more than $22 \mathrm{~g}$. Flunixin concentrations remained detectable, but were below inferred

255 therapeutic concentrations, for 36-40 h after consumption of the flunixin supplemented feed

256 ceased (Figure 1). Currently there are no toxicity data for flunixin in sheep, however the animals

257 used in this study did not show any visible side effects as a result of consuming flunixin

258 supplemented feed.

259 Previous work in cattle by Odensvik, $(1995 ; 1998)$ showed that oral administration of flunixin

$260(2.2 \mathrm{mg} / \mathrm{kg})$ as a granule inhibited the production of prostaglandin $\mathrm{PGF}_{2} \alpha$ by up to $60 \%$, which

261 was as effective as the standard therapeutic dose of flunixin $(2.2 \mathrm{mg} / \mathrm{kg})$ used parenterally. 
262 Although the authors did not directly measure the effectiveness of oral flunixin at reducing

263 inflammation, they concluded that an anti-inflammatory effect was likely to occur due to reduced

264 production of $\mathrm{PGF}_{2} \alpha$ which acts as a pro-inflammatory factor following injury (Ricciotti \&

265 FitzGerald, 2011). Although further studies are required it is expected that oral administration of

266 flunixin could provide effective pain relief in sheep.

267 In conclusion, results of this study demonstrate that when flunixin is administered orally through

268 feed to sheep it is absorbed rapidly into the bloodstream and despite variability in consumption

269 rates of feed, all sheep reached inferred therapeutics concentrations of flunixin within $6 \mathrm{~h}$ of

270 starting to consume the feed. Further studies are required to investigate potential binding of

271 flunixin to various feed components and potential impacts that such binding may have on

272 toxicity. The possible mild aversion to feed supplemented with flunixin on day 1 did not persist

273 on day 2 indicating that the medicated feed is readily accepted by sheep. Supplementation of

274 feed with flunixin may provide a practical way to provide pain relief to sheep prior to and after

275 painful husbandry procedures thus eliminating the need for multiple injections, reducing

276 handling stress and minimising labour requirements.

\section{ACKNOWLEDGEMENTS}

278 We thank Sue Belson, Brad Hine, Dominic Niemeyer, Tim Dyall, Aurélie Bussy and Etienne

279 Goumand for their assistance during the experiment. We also thank Paul Mills (The University

280 of Queensland) for his advice on pharmacokinetic analyses. 
282 Chapple RS, Wodzicka-Tomaszewska M, Lynch JJ. 1987. The learning behaviour of sheep when

283 introduced to wheat. I. Wheat acceptance by sheep and the effect of trough familiarity. Applied

284 Animal Behaviour Science 18:157-162. http://dx.doi.org/10.1016/0168-1591(87)90189-4

285 Cheng Z, McKeller Q, Nolan A. 1998a. Pharmacokinetic studies of flunixin meglumine and

286 phenylbutazone in plasma, exudate and transudate in sheep. Journal of Veterinary Pharmacology

287 and Therapeutics 21:315-321. 10.1046/j.1365-2885.1998.00144.x

288 Cheng Z, Nolan AM, McKellar QA. 1998b. Measurement of cyclooxygenase inhibition in vivo:

289 A Study of Two Non-Steroidal Anti-Inflammatory Drugs in Sheep. Inflammation 22:353-366.

$29010.1023 / \mathrm{a}: 1022364731126$

291 Coetzee JF, Edwards LN, Mosher RA, Bello NM, O'Connor AM, Wang B, KuKanich B, Blasi

292 DA. 2012. Effect of oral meloxicam on health and performance of beef steers relative to bulls 293 castrated on arrival at the feedlot. Journal of Animal Science 90:1026-1039. 10.2527/jas.2011$294 \quad 4068$

295

296

297

298

299

300

301

302

Currah JM, Hendrick SH, Stookey JM. 2009. The behavioral assessment and alleviation of pain associated with castration in beef calves treated with flunixin meglumine and caudal lidocaine epidural anesthesia with epinephrine. The Canadian Veterinary Journal 50:375-382.

Hinch GN, Hills J, Lynch JJ, Nolan JV. 2004. Familiar odour and flavour cues reduce feed neophobia in sheep. Animal Production in Australia 1:97-99.

http://dx.doi.org/10.1071/SA0401025

Keegan KG, Messer NT, Reed SK, Wilson DA, Kramer J. 2008. Effectiveness of administration of phenylbutazone alone or concurrent administration of phenylbutazone and flunixin meglumine 
303 to alleviate lameness in horses. American Journal of Veterinary Research 69:167-173.

304 10.2460/ajvr.69.2.167

305 Königsson K, Törneke K, Engeland IV, Odensvik K, Kindahl H. 2003. Pharmacokinetics and

306 pharmacodynamic effects of flunixin after intravenous, intramuscular and oral administration to 307 dairy goats. Acta Veterinaria Scandinavica 44:153-159.

308 Launchbaugh KL, Provenza FD, Werkmeister MJ. 1997. Overcoming food neophobia in

309 domestic ruminants through addition of a familiar flavor and repeated exposure to novel foods.

310 Applied Animal Behaviour Science 54:327-334. http://dx.doi.org/10.1016/S0168-

311 1591(96)01194-X

312 Lizarraga I, Chambers JP. 2012. Use of analgesic drugs for pain management in sheep. New

313 Zealand Veterinary Journal 60:87-94. 10.1080/00480169.2011.642772

314 Marini D, Pippia J, Colditz IG, Hinch G, Petherick JC, Lee C. 2015. Randomised trial of the 315 bioavailability and efficacy of orally administered flunixin, carprofen and ketoprofen in a pain 316 model in sheep. Australian Veterinary Journal 93:265-270. 10.1111/avj.12351

317 Mosher RA, Coetzee JF, Cull CA, Gehring R, KuKanich B. 2012. Pharmacokinetics of oral 318 meloxicam in ruminant and preruminant calves.. Journal of Veterinary Pharmacology and 319 Therapeutics 35:373-381. 10.1111/j.1365-2885.2011.01331.x

320 Odensvik K. 1995. Pharmacokinetics of flunixin and its effect on prostaglandin F metabolite 321 concentrations after oral and intravenous administration in heifers. Journal of Veterinary 322 Pharmacology and Therapeutics 18:254-259. 10.1111/j.1365-2885.1995.tb00589.x 
323 Paull DR, Lee C, Colditz IG, Atkinson SJ, Fisher AD. 2007. The effect of a topical anaesthetic

324 formulation, systemic flunixin and carprofen, singly or in combination, on cortisol and

325 behavioural responses of Merino lambs to mulesing. Australian Veterinary Journal 85:98-106.

$326 \quad 10.1111 / \mathrm{j} .1751-0813.2007 .00115 . \mathrm{x}$

327 Pinheiro J, Bates D, DebRoy S, Sarkar D, R Core Team. 2015. nlme: Linear and nonlinear mixed

328 effects models. R package version 3.1-121, http://CRAN.R-project.org/package=nlme.

329 Pellegrini-Masini A, Poppenga RH, Sweeney RW. 2004. Disposition of flunixin meglumine

330 injectable preparation administered orally to healthy horses. Journal of Veterinary

331 Pharmacology and Therapeutics 27:183-186. 10.1111/j.1365-2885.2004.00575.x

332 Ricciotti E, FitzGerald GA. 2011. Prostaglandins and inflammation. Arteriosclerosis,

333 Thrombosis, and Vascular Biology 31:986-1000. 10.1161/atvbaha.110.207449

334 Toutain PL, Autefage A, Legrand C, Alvinerie M. 1994. Plasma concentrations and therapeutic

335 efficacy of phenylbutazone and flunixin meglumine in the horse:

336 pharmacokinetic/pharmacodynamic modelling. Journal of Veterinary Pharmacology and

337 Therapeutics 17:459-469. 10.1111/j.1365-2885.1994.tb00278.x

338 Welsh EM, Nolan AM. 1995. Effect of flunixin meglumine on the thresholds to mechanical

339 stimulation in healthy and lame sheep. Research in Veterinary Science 58:61-66. 10.1016/0034-

$340 \quad 5288(95) 90090-\mathrm{x}$

341 Welsh EM, McKellar QA, Nolan AM. 1993. The pharmacokinetics of flunixin meglumine in the

342 sheep. Journal of Veterinary Pharmacology and Therapeutics 16:181-188. 10.1111/j.1365-

$343 \quad 2885.1993 . t b 00162 . x$ 
344 Welsh JCM, Lees P, Stodulski G, Cambridge H, Foster AP. 1992. Influence of feeding schedule 345 on the absorption of orally administered flunixin in the horse. Equine Veterinary Journal 24:62$346 \quad 65.10 .1111 / \mathrm{j} .2042-3306.1992 . t b 04776 . x$

347 Zang Y, Huo M, Zhou J, Xie S. 2010. PKSolver: An add-in program for pharmacokinetic and 348 pharmacodynamic data analysis in Microsoft Excel. Computer Methods and Programs in 349 Biomedicine 99:306-314. 
351 Table 1: Palatability test results (mean \pm S.D.) for the effect of interaction of feed type (flunixin supplemented or control) by day (1 or

\begin{tabular}{|c|cc|cc|}
\hline & \multicolumn{2}{|c|}{ Day 1 } & \multicolumn{2}{c|}{ Day 2 } \\
\hline Location & Control & Flunixin supplemented & Control & Flunixin supplemented \\
\hline Left & $906.00 \pm 426.28$ & $451.75 \pm 338.76$ & $707.88 \pm 451.40$ & $590.67 \pm 518.79$ \\
Right & $1158.88 \pm 330.73$ & $562.83 \pm 358.93$ & $364.50 \pm 446.95$ & $561.63 \pm 309.77$ \\
\hline Mean & $1050.50 \pm 365.42$ & $499.36 \pm 348.68^{\mathrm{A}}$ & $560.71 \pm 449.62^{\mathrm{A}}$ & $574.07 \pm 414.19$ \\
\hline
\end{tabular}




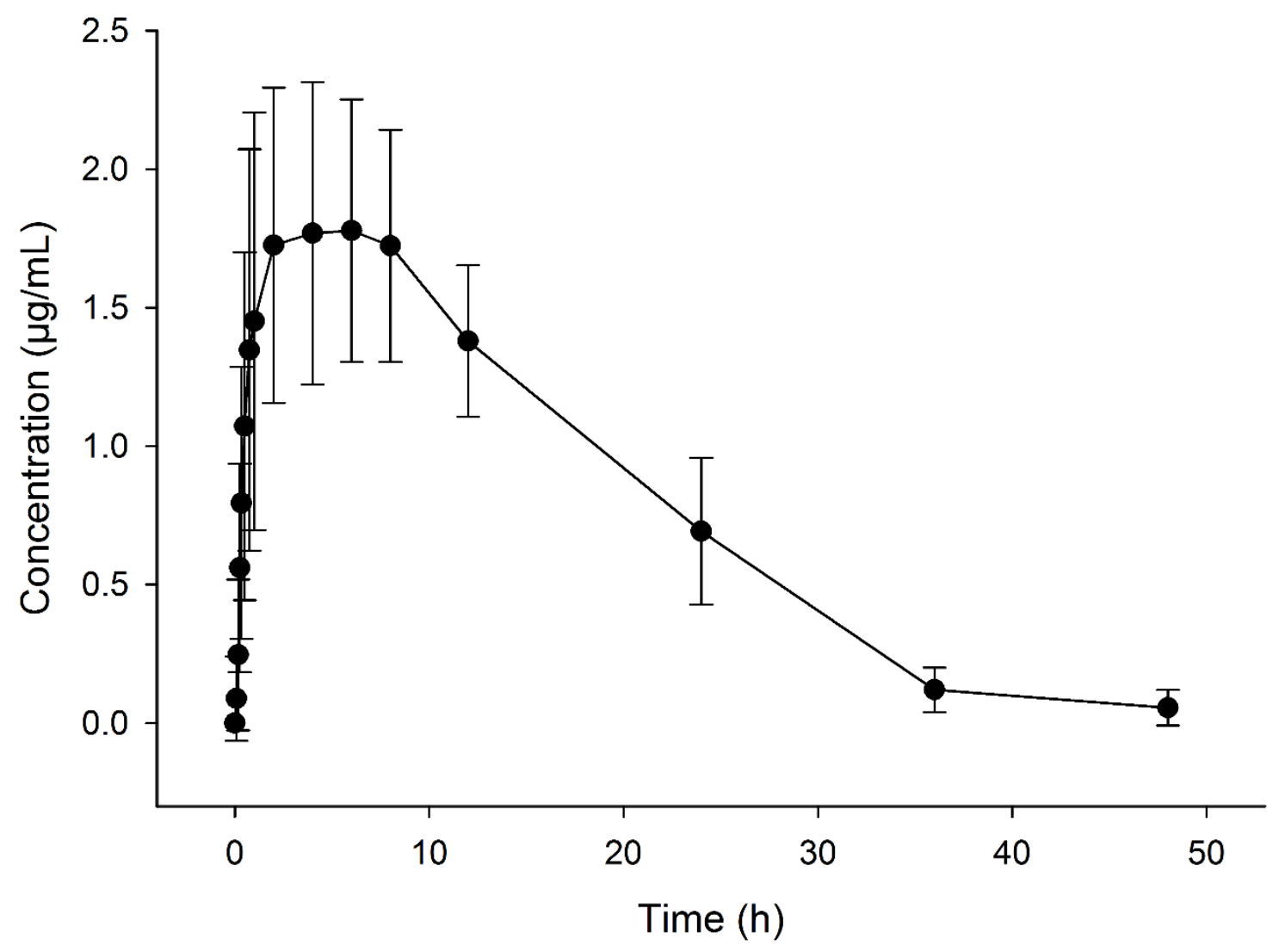

Figure 1: Flunixin in plasma concentration time curve (means \pm S.D.) of eight sheep over a $48 \mathrm{~h}$ period following administration of flunixin $(4.0 \mathrm{mg} / \mathrm{kg})$ through pelleted feed. 


\begin{tabular}{|c|c|c|c|}
\hline Time feed was weighed $(\mathrm{h})$ & Average intake $(\mathrm{g}) \pm$ S.D. & Median $(\mathrm{g})$ & Range $(\mathrm{g})$ \\
\hline 0.08 & $174.69 \pm 112.12$ & 205.75 & $21.50-357.50$ \\
0.17 & $26.25 \pm 29.66$ & 18.50 & $0.00-71.00$ \\
0.25 & $18.63 \pm 16.01$ & 15.00 & $0.00-48.00$ \\
0.33 & $6.06 \pm 12.27$ & 1.00 & $0.00-36.00$ \\
0.50 & $23.44 \pm 17.30$ & 25.00 & $0.00-50.50$ \\
0.75 & $13.13 \pm 20.79$ & 6.50 & $0.00-62.50$ \\
1 & $6.19 \pm 15.72$ & 0.00 & $0.00-45.00$ \\
2 & $91.38 \pm 58.90$ & 75.25 & $32.00-211.00$ \\
4 & $151.63 \pm 39.12$ & 148.25 & $89.00-220.00$ \\
6 & $141.56 \pm 56.38$ & 149.75 & $68.00-211.00$ \\
8 & $88.38 \pm 59.54$ & 71.25 & $31.00-194.00$ \\
12 & $58.69 \pm 114.15$ & 8.50 & $0.00-332.50$ \\
\hline
\end{tabular}


374 Table 3: Flunixin pharmacokinetic parameters following oral administration through $800 \mathrm{~g}$ of pelleted feed to eight sheep at a dose

\begin{tabular}{|c|c|c|c|c|c|c|c|c|c|}
\hline \multirow[b]{2}{*}{ Parameter (units) } & \multicolumn{9}{|c|}{ Sheep ID } \\
\hline & 305 & 466 & 580 & 612 & 621 & 627 & 648 & 732 & Mean \pm S.D. \\
\hline $\mathrm{t}_{1 / 2}, \mathrm{~h}$ & 4.59 & 5.39 & 8.23 & 6.29 & 7.31 & 4.85 & 11.04 & 5.19 & $7.95 \pm 2.19$ \\
\hline Tmax, h & 8.00 & 1.00 & 6.00 & 6.00 & 2.00 & 12.00 & 12.00 & 4.00 & $6.00 \pm 4.14$ \\
\hline $\mathrm{Cmax}, \mu \mathrm{g} / \mathrm{mL}$ & 2.39 & 1.61 & 2.18 & 1.89 & 2.16 & 1.33 & 1.63 & 2.80 & $1.78 \pm 0.48$ \\
\hline AUC0-t, $\mu \mathrm{g} / \mathrm{mL}^{*} \mathrm{~h}$ & 29.96 & 38.00 & 38.21 & 40.99 & 42.78 & 31.84 & 42.75 & 36.05 & $37.68 \pm 4.77$ \\
\hline MRT, h & 9.36 & 14.34 & 13.36 & 13.43 & 12.98 & 15.80 & 19.48 & 9.32 & $13.59 \pm 3.31$ \\
\hline
\end{tabular}

